\title{
Écrire dans la variante de l'autre : le cas de Sous les vents de Neptune de Fred Vargas
}

\section{Nadine Vincent}

\section{OpenEdition \\ Journals}

Édition électronique

URL : http://journals.openedition.org/coma/317

DOI : $10.4000 /$ coma.317

ISSN : 2275-1742

Éditeur

Institut des textes \& manuscrits modernes (ITEM)

\section{Référence électronique}

Nadine Vincent, «Écrire dans la variante de l'autre : le cas de Sous les vents de Neptune de Fred Vargas », Continents manuscrits [En ligne], 2 | 2014, mis en ligne le 22 avril 2014, consulté le 23 avril 2019. URL : http://journals.openedition.org/coma/317 ; DOI : 10.4000/coma.317

Ce document a été généré automatiquement le 23 avril 2019

\section{(i)

Continents manuscrits - Génétique des textes littéraires - Afrique, Caraîbe, dispora est mis à disposition selon les termes de la licence Creative Commons Attribution - Pas d'Utilisation

Commerciale - Pas de Modification 4.0 International. 


\title{
Écrire dans la variante de l'autre : le cas de Sous les vents de Neptune de Fred Vargas
}

\author{
Nadine Vincent
}

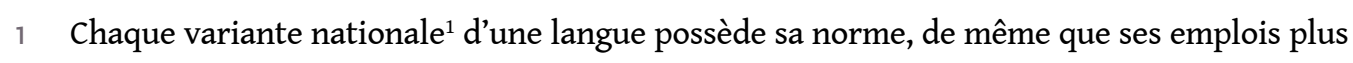
familiers. Si la norme est davantage soumise à la description des ouvrages de référence, c'est dans la langue familière, elle aussi régie par ses propres règles, que l'on goûte toute la vitalité d'une culture.

Les écrivains francophones hors de France ${ }^{2}$ sont confrontés très tôt à la variation linguistique et à cette cohabitation de systèmes hiérarchisés qu'ils doivent maîtriser pour communiquer efficacement.

2 Les écrivains francophones ${ }^{3}$ reçoivent [...] en partage une sensibilité plus grande à la problématique des langues, sensibilité qui s'exprime par de nombreux témoignages attestant à quel point l'écriture, pour chacun d'eux, est synonyme d'inconfort et de doute. La notion de surconscience renvoie à ce que cette situation d'inconfort peut avoir d'exacerbé et de fécond. ${ }^{4}$

3 Moins en contact avec d'autres normes du français que la norme hexagonale, comment réagissent les auteurs français quand ils écrivent dans une variante qui n'est pas la leur? Nous nous intéressons ici au cas de Fred Vargas, qui a situé une partie de l'action de son roman Sous les vents de Neptune dans l'Outaouais québécois et dont les dialogues en français québécois ont fait couler beaucoup d'encre. Après avoir recensé quelques critiques du roman provenant d'un côté et de l'autre de l'Atlantique, nous présenterons les explications de l'auteure et examinerons sous un angle linguistique quelques-uns des traits caractéristiques du français québécois utilisé dans son roman. Ce tour d'horizon nous permettra de réfléchir à la hiérarchisation des variantes en fonction du point d'ancrage des locuteurs. 


\section{Qui est Fred Vargas}

4 Fille d'un intellectuel et d'une scientifique, Fred Vargas, de son vrai nom Frédérique Audoin-Rouzeau, est née à Paris en 1957. Docteure en archéologie médiévale, chercheuse au CNRS, elle se spécialise en archéozoologie ${ }^{5}$. «Parallèlement à ses activités littéraires, elle poursuivra donc une brillante carrière scientifique. À moins que ce ne soit l'inverse. ${ }^{6}$ »

En se basant sur cette carrière scientifique, on attribue volontiers à Fred Vargas une précision, une rigueur, un sens des détails qui transparaîtrait dans son travail de romancière, notamment dans l'attention qu'elle porte à la langue :

Ses talents de fouineuse scientifique, elle les a tout naturellement transposés dans des rom'pol' qui sont un exigeant violon d'Ingres. Fred Vargas écrit ses romans très vite, un premier jet en trois semaines de vacances, mais elle passe ensuite des mois à corriger son manuscrit, mettant un soin d'orfèvre à choisir ses mots tant pour leur justesse que pour leur musique. ${ }^{7}$

\section{Intrigue et contexte}

En 2004, l'auteure à succès ${ }^{8}$ publie Sous les vents de Neptune, son neuvième roman policier. Dans ce roman, son personnage principal, le commissaire Jean-Baptiste Adamsberg, visite l'Outaouais québécois avec sa brigade parisienne pour suivre un stage sur les empreintes génétiques dans les bureaux de la Gendarmerie royale du Canada (police fédérale). Ce contexte amène la création de plusieurs personnages québécois (policiers, hôtelier, barman, photographe), et permet à l'auteure d'écrire des dialogues colorés, qui mettent en interaction deux Québécois, ou un Français et un Québécois, avec tous les malentendus que l'on peut imaginer.

\section{La réception critique en Europe et au Québec}

Comme pour chacun des romans précédents de Fred Vargas, la réception européenne de Sous les vents de Neptune est enthousiaste. «Sous les vents de Neptune est attendu comme le messie par la presse et le public. [...] La revue 813 les amis des littératures policières témoigne une fois encore de son soutien à Fred Vargas en lui décernant le trophée 813 du meilleur roman francophone 2004. ${ }^{9}$ "

Chaque fois qu'on évoque la langue attribuée aux locuteurs québécois, c'est pour en souligner la justesse, la qualité, la force d'évocation.

Le séjour au Québec de l'équipe d'Adamsberg permet aussi à Vargas d'ajouter à sa langue déjà riche les expressions savoureuses d'un français différent, imagé, truculent. ${ }^{10}$

Dialogues enlevés et percutants: le langage québécois, jamais gratuit, jamais caricatural, apporte sa part d'étrangeté. ${ }^{11}$

On n'en dira pas plus de cette course du limier à travers les brumes de la Belle Province, dont accents et vocabulaire donnent au livre son parler-vrai. ${ }^{12}$

La romancière, à l'évidence, s'amuse elle-même en cherchant à nous séduire, amuser, et réussit merveilleusement son coup, une fois de plus. Le parler québécois venant, ici, comme un nappage de sirop d'érable sur le gâteau. Goûteux $!^{13}$

Cette belle unanimité ne traverse cependant pas l'océan Atlantique, à la grande surprise des Français : «On a du mal à le croire, mais il existe une région du monde où l'un des 
romans de Fred Vargas a été accueilli tièdement. La Belle Province n’a toujours pas digéré les écarts de langage de Sous les vents de Neptune. ${ }^{14}$ "

8 Si les commentateurs européens se régalent de la langue québécoise illustrée par Vargas, c'est tout le contraire pour les Québécois qui s'insurgent contre l'image du Québec et de sa langue dans Sous les vents de Neptune, se demandant comment une telle méconnaissance est encore possible au xxI ${ }^{\mathrm{e}}$ siècle. Les critiques prennent les lecteurs à témoin en donnant de nombreux exemples :

«Tais ton bec, le Français. Mouve-toi d'ici ou j'appelle les cochs.» Cette phrase colorée semble tout droit sortie d'un roman ayant la banlieue parisienne comme toile de fond. Or il n'en est rien. Il s'agit plutôt d'une réplique lancée par un barman de Gatineau, $\mathrm{PQ}$, dans le plus récent roman policier de l'écrivaine française Fred Vargas. «Ta yeule, le Franças. Câlice ton camp ou je call les bœufs », aurait sonné plus vrai. ${ }^{15}$

Et ils parlent, mes amis, ils parlent. « Tu crois-tu que c'est lequel, le commissaire ?» « Pas trop ébarroui par le voyage ? » [...] « Il est boqué comme un ours.» « Tais ton bec, le Français. » « Faut qu'on se mouve. Si le boss nous pogne à brasser de l'air, il va manger ses bas. » «Criss, on va pas javasser des heures. » [...] je vous en conjure, si vous connaissez quelqu'un qui parle comme ça, dites-lui de m'écrire, je publierai son portrait. ${ }^{16}$

Archéozoologue médiéviste, Fred Vargas est pourtant reconnue pour le soin méticuleux dont elle fait preuve, habituellement, dans ses recherches. Force est d'admettre que cette fois, elle est complètement à côté de ses pompes, comme disent les Français. Et qu'elle vient de rater une belle occasion de se taire le bec... ${ }^{17}$ On se souviendra que dans le neuvième rompol, Sous les vents de Neptune, le commissaire Adamsberg avait failli mourir dans les boisés de Gatineau au Québec. Et que les lecteurs et lectrices québécois avaient de leur côté failli mourir de rire en découvrant une nouvelle version de leur langue, inventée par $\mathrm{M}^{\mathrm{me}}$ Vargas. Certains ont moins ri que d'autres, ils en ont même voulu à l'auteure. ${ }^{18}$

Comment expliquer une telle divergence entre les appréciations des Européens et des Québécois? Pour que les premiers y croient, il faut que les traits reproduits soient un écho crédible du français en usage au Québec. Pour que les seconds réagissent aussi épidermiquement, il faut que les erreurs soient nombreuses et frappantes. Autrement dit, la cible visée était la bonne, mais elle n'a pas été atteinte.

\section{Fred Vargas, le Québec et le français québécois}

On peut supposer que les écrivains qui s'attaquent au défi d'écrire dans une variante qui n'est pas la leur ont déjà une bonne connaissance de cette variante. Était-ce le cas pour Fred Vargas? Quel était son lien avec le Québec? Et pourquoi l'Outaouais? Les raisons apparaissent toutes simples. Fort probablement parce qu'elle a rendu visite à un de ses bons amis, l'illustrateur Edmond Baudoin, qui a enseigné à l'université du Québec en Outaouais (UQO) de 1999 à 2003 ${ }^{19}$, justement à Gatineau, ville où séjourne la brigade parisienne dans Sous les vents de Neptune.

Fred Vargas évoque ses deux séjours au Québec. "J'ai été tellement captivée par leurs expressions, explique-t-elle, que j'ai acheté un carnet où je notais tout: de «Tu veux-tu? ", « chum » pour «mec » à "soulever mer et monde », en passant par la jolie formule «pelleteux de nuages » qui convient si bien à Adamsberg. J'ai tellement aimé ça que j'en ai un peu rajouté. C'est pour cette raison, entre autres, que j'ai dû amputer ce roman de cent pages. ${ }^{20}$ "

C'est donc vraisemblablement après seulement deux séjours au Québec, et avec une vision très impressionniste de la langue qui y est parlée, que Fred Vargas s'est lancée dans 
l'écriture de dialogues en français québécois. Elle ne réagira pas immédiatement aux reproches émis par les Québécois, mais tentera de se justifier en 2006, au moment de la sortie de son roman suivant, Dans les bois éternels :

Je saisis l'occasion de dire ici ce que je souhaitais dire à tous les Québécois depuis la sortie de Sous les vents de Neptune, et du malentendu linguistique qui s'ensuivit: je savais pertinemment, en l'écrivant, que j'inventais du "faux québécois", compressant et tordant la langue. Je le savais si bien que je n'ai pas fait relire mon manuscrit à mes amis de Hull, car je devinais très bien leur censure ! [...] J'ai fait erreur, donc, et que les Québécois veuillent bien m'en excuser. Qu'ils sachent que cette liberté prise avec leurs mots n'allait nullement à leur encontre, tout au contraire. $^{21}$

11 L'explication donnée est plutôt mince. D'abord, pourquoi avoir attendu deux ans avant de réagir? Et ensuite, que veut dire «inventer du faux québécois » en «compressant et tordant la langue»? Il aurait été utile que Fred Vargas donne quelques détails sur ses procédés créatifs de manière à ce que les Québécois soient en mesure de juger par euxmêmes. Faute d'explications satisfaisantes, examinons sous un angle linguistique quelques-unes des caractéristiques les plus évidentes de cette nouvelle langue québécoise.

\section{Quelques éléments linguistiques}

Les particularités linguistiques de la langue «créée » par Fred Vargas sont multiples. Nous nous pencherons ici sur trois éléments très présents dans le roman et qui ont particulièrement fait réagir les Québécois : a) la variation diastratique ; b) l'emploi de la particule interrogative « -tu »; c) le vocabulaire et les expressions.

\section{Sociolinguistique et impérialisme linguistique}

13 La première trace d'ignorance face à la notion de variation linguistique est de penser qu'il existe une norme, celle de France, et que toutes les autres variétés n'existent que pour la langue familière. Fred Vargas a misé juste en faisant dîner les Québécois le midi, en les faisant souper le soir, ou en mettant le mot «courriel» dans la bouche d'un policier québécois, ce qui respecte la norme du français au Québec. Outre quelques exceptions, elle a cependant généralement supposé que tous les personnages québécois ne parlaient qu'en langue familière, quelle que soit la situation de communication. Ainsi, qu'un lieutenant québécois, lors d'une réception à Paris, dise à un collègue français : «Criss, ça fait plaisir de voir ça [...]. Tout le monde est habillé fouledresse, hein ? T'es bien beau sur ton forty-five. ${ }^{22}$ " apparaît pour le moins invraisemblable. Et, bien sûr, comme les Québécois ne parlent qu'en langue familière, les Français, eux, ne parlent qu'en langue standard. Ainsi, un Québécois qui rencontre Adamsberg dans un sentier devine qu'il est Français. «Comment je le sais? dit l'homme en riant [...]. Parce que quand tu parles, je crois pas t'entendre, je crois te lire..$^{23}$ "

De là à supposer que les Français sont des êtres civilisés venant séjourner dans une peuplade de sympathiques arriérés, il n'y a qu'un pas que Vargas ne voulait certainement pas franchir, mais qu'elle n'a pu éviter en tombant dans des clichés gros comme des pièges à ours: "L'homme, taillé comme un bûcheron canadien dans les livres d'images, contourna son bar et souleva Adamsberg par les aisselles ${ }^{24}$ ». C'est le genre de stéréotype qui, pour un lecteur québécois, ne passe pas inaperçu, surtout quand il se répète page 
après page. Cette vision folklorisante du Québec a été abondamment dénoncée dans les journaux québécois.

Il faut voir l'équipe de choc du module spécialisé en «empreintes génétiques » qui les accueille à leur arrivée au Québec, tous affublés de prénoms savoureux et révolus : Aurèle, Berthe, Philibert, Alphonse, Ginette et Fernand. Une escouade de septuagénaires édentés ? [...] En outre, Fred Vargas fonce tête baissée dans les habituels clichés "canadiens». Les grands espaces, le froid (les routes non asphaltées à $50 \mathrm{~km}$ de la capitale fédérale, «attendu que le gel s'employait à faire exploser l'asphalte chaque hiver »), les colifichets à rapporter aux copains qui vont de la «bonne grosse veste d'intérieur en peau d'ours à carreaux » aux « bottines en poil d'élan $\aleph^{25}$.

On dit que Fred Vargas a séjourné en Outaouais, dans la région de Gatineau. Elle en a rapporté une image caricaturale des Québécois, bourrée de stéréotypes dignes de l'époque de Voltaire. À l'en croire, les écureuils circulent aussi librement dans la ville que les voitures, et les indigènes s'expriment dans un langage pour le moins « pittoresque » [...]. On se croirait dans un camp de bûcherons des années $1950 !^{26}$

\section{La particule interrogative « -tu »}

La forme interrogative en «-tu », caractéristique de la langue orale familière au Québec, correspond à son équivalent en «-ti » en France rurale. Vous venez-ti ? Vous venez-tu?

Fred Vargas a vu juste en utilisant ce trait propre au français québécois, mais elle en a généralisé l'usage, sans tenir compte des critères qui dictent son application.

- La question doit être directe (on y répond par oui ou par non).

- La particule «-tu » marquant à elle seule l'interrogation, elle ne peut pas être utilisée s'il y a inversion du sujet ou présence d'un adverbe interrogatif.

- La particule «-tu» ne peut apparaître dans une proposition interrogative indirecte (introduite par la conjonction si ou par un terme interrogatif: pronom, déterminant ou adverbe).

- À moins de vouloir produire un effet particulier, la particule «-tu » n'est pas utilisée avec une formulation négative.

Voici quelques-uns des nombreux exemples de questions mises dans la bouche de Québécois par Fred Vargas qui ne répondent pas à ces critères :

« Tu crois-tu que c'est lequel, le commissaire ? ${ }^{27}$ »

" Pourquoi tu portes-tu deux montres $?^{28}$ "

" Ça te gêne-tu pas que je m'assoie ? ${ }^{29}$ "

"Tu vas-tu faire quoi, ce soir ? ${ }^{30} "$

«Cette combinaison [...], si tu te souviens-tu, constitue son profil génétique ${ }^{31}$.»

"Comment tu le sais-tu ?32"

Au Québec comme ailleurs, ces questions auraient dû se formuler ainsi :

- Tu crois que c'est lequel, le commissaire?

- Pourquoi tu portes deux montres? (ou Pourquoi portes-tu deux montres ?)

- Ça te gêne que je m'assoie ? (ou Est-ce que ça te gêne que je m'assoie ?)

- Tu vas faire quoi, ce soir? (ou Que vas-tu faire, ce soir ?)

- Cette combinaison, si tu te souviens, constitue son profil génétique.

- Comment tu le sais ? (ou Comment le sais-tu ?) 


\section{Vocabulaire et expressions}

16 Les critiques québécois ont cité de nombreux mots et expressions qui leur semblaient incorrects ou inconnus. Il n'est évidemment pas possible pour chaque Québécois de connaître l'ensemble des emplois en usage sur le territoire. Pour mesurer la vigueur d'un mot ou d'une expression, nous avons sondé le corpus journalistique Eureka, qui recense une grande quantité de publications (articles parus dans des quotidiens, des magazines, communiqués d'agence de presse, etc.) en Europe et au Canada. Nous avons également consulté des sources lexicographiques québécoises spécialisées pour vérifier l'existence de quelques-uns de ces usages.

\section{Ébarrouir ou ébarouir}

« Bienvenue, commissaire principal. Pas trop ébarroui par le voyage ? ${ }^{33}$ »

« T'as l'air ébarroui. ${ }^{34}$ "

Selon les dictionnaires consultés, l'emploi du verbe éba(r)rouir est vieilli. On en trouve une dizaine d'occurrences dans les journaux, notamment dans Midi-Libre, pour mentionner la parution en Suisse d'un dictionnaire des verbes oubliés, ou dans des journaux québécois ou acadiens pour souligner des emplois anciens utilisés par des écrivains. Par ailleurs, on ne trouve que trois attestations de la graphie avec deux $r$ dans les journaux. L'une apparait en 1997 dans La Croix, où l'on souligne l'usage de cet adjectif dans un roman de l'Acadienne Antonine Maillet, l'autre est attestée en 2001 dans Sud Ouest, dans un article où l'on reproduit le français régional de Charente, et la troisième se retrouve sous la plume d'un journaliste québécois critiquant le roman de Fred Vargas. Ébarouir et ébaroui, avec un seul « $\mathrm{r} »$, sont attestés dans la plupart des glossaires et dictionnaires québécois. Avec deux $r$, ainsi que l'écrit Vargas, on ne retrouve que l'adjectif, et seulement dans un corpus d'œuvres littéraires publié au début des années 1980 par des chercheurs de l'université de Montréal ${ }^{35}$, auquel il serait étonnant que l'auteure ait eu accès. Chose certaine, ce mot n'est plus en usage au Québec.

\section{Être tendre d'entretien}

«Et toi ? Avec qui travailles-tu ? - Avec celle qui est tendre d'entretien. Tu peux-tu me rappeler son nom? - Tendre d'entretien? - Grosse, traduisit Sanscartier, embarrassé. ${ }^{36}$ "

Absent des contextes journalistiques, l'expression "être tendre d'entretien» est seulement attestée dans le très critiqué Dictionnaire de la langue québécoise de Léandre Bergeron $^{37}$ : « Être tendre d'entretien - avoir de l'embonpoint. ». C'est donc une expression d'origine inconnue et de fréquence nulle.

\section{Javasser}

"Criss, s'énerva le barman, on va pas javasser des heures. ${ }^{38}$ "

On trouve trois attestations du verbe javasser dans les journaux. Une dans un article du Point qui cite un jardinier saintongeais, et deux dans des articles québécois qui citent le roman de Fred Vargas. Le verbe est cependant attesté dans le sens de «bavarder », dans quelques glossaires québécois du début du xxe siècle (Dionne, 1909, Glossaire du parler français au Canada, 1930) et dans le dictionnaire de Léandre Bergeron (1980). Visiblement, il est aujourd'hui sorti de l'usage au Québec. 


\section{Prendre la quille (de l'air)}

«Il a pris la quille de l'air, votre boss ! $!^{39}$ "

«T'as toujours pas compris qu'il a pris la quille ? ${ }^{40}$ "

Cette expression, qui n'apparaît dans le corpus journalistique que dans un article qui critique le roman de Fred Vargas, est consignée dans deux ouvrages de Victor Barbeau. Dans Le Ramage de mon pays, paru en 1939, il indique : «Prendre la quille de l'air - Laisser quelqu'un en plan. ». Dans une refonte paru en 1963, il indique plutôt : "Prendre la quille de l'air - loc. Être congédié.». L'expression n'a donc jamais été courante, et est aujourd'hui tout à fait inconnue des Québécois.

\section{Taire son bec}

«Tais ton bec, le Français. ${ }^{41}$ »

"Après, il n'y a plus moyen de leur faire taire le bec..$^{42}$ "

"Je sais taire mon bec, commissaire. Et je suis moins dangereux que vous. ${ }^{43}$ "

L'expression « taire son bec » est absente du corpus Eureka, si on fait abstraction des trois articles de journalistes québécois qui critiquent le roman de Fred Vargas. Cependant, l'expression est attestée dans le glossaire de Narcisse-Eutrope Dionne, sous bec: "Taire son bec, cesser de parler» (p.67) et sous taire: "Taire son bec, taire sa gueule: se taire, garder un secret»(p.623). Ce glossaire a été publié en 1909 et réimprimé en 1974. L'expression était donc peu répandue et a aujourd'hui disparu.

Nous pourrions multiplier les exemples, mais ces quelques cas suffisent à illustrer deux choses:

- Fred Vargas n'a pas inventé une langue québécoise, elle a seulement eu recours à des emplois vieillis ou marginaux, probablement sans savoir qu'il ne s'agissait pas d'emplois courants au Québec.

- Fred Vargas n'a vraisemblablement pas pu consulter les sources lexicographiques, parfois anciennes et difficiles d'accès, qui attestaient les emplois qu'elle a retenus.

La question qui se pose est donc la suivante: sur quoi s'est basée l'auteure pour construire la langue québécoise de son roman? Une hypothèse a été émise par le chercheur Pierre Larrivée en 2006 : « une partie des erreurs de Vargas sont apparemment dues aux indications inexactes données par le Dictionnaire des expressions québécoises de Pierre Desruisseaux ${ }^{44} »$.

19 Ce « dictionnaire ", qui compile sans les distinguer des mots et expressions puisés à même des sources d'époques différentes et de fiabilités variables, n'est pas recommandable d'un point de vue lexicographique, mais constitue un succès de librairie au Québec, probablement en raison de son contenu pittoresque. C'est le genre de livre de poche qu'achètent les touristes en quête d'exotisme en espérant rapporter avec eux un peu de la langue colorée qui les a charmés lors de leur passage au Québec. Nous avons retrouvé chez Desruisseaux les expressions et mots présentés plus haut, et la bibliographie de son dictionnaire atteste toutes les sources lexicographiques plus spécialisées que nous avons consultées.

Qu'une auteure, doublée d'une scientifique, se contente d'une telle source pour penser reproduire une variété de langue qui n'est pas la sienne témoigne d'une méconnaissance évidente de la variation linguistique. 


\section{L'histoire se répète}

21 Cette histoire n'est pas unique. Que des auteurs français décident de décrire le français en usage au Québec, ou les réalités québécoises, sans consulter les principaux concernés, fait partie de la relation historique conflictuelle entre le Québec et la France. En 1997, le Bescherelle décidait d'ouvrir les pages de son guide de conjugaison aux verbes de la francophonie. «Pour la première fois dans un manuel de conjugaison, figurent également des verbes spécifiques à la Belgique, au Canada, à l'Afrique francophone.» (Bescherelle, 1997 : avant-propos). Les verbes canadiens, marqués « québécois » dans l'ouvrage, avaient tous été extraits du Dictionnaire de la langue québécoise de Léandre Bergeron, qui a recensé sans distinction des emplois vieillis, des régionalismes, de l'oral urbain, sans compter plusieurs mots et expressions d'origine inconnue :

Ce dictionnaire a été vivement critiqué lors de sa parution et semblait avoir été relégué aux oubliettes. Or, c'est précisément cet ouvrage que le réputé linguiste Michel Arrivé a approuvé pour faire place, dans les listes bescherelliennes, à la langue québécoise; et ce, sans qu'il y ait consultation, du moins apparemment, de nos instances, comme, entre autres, l'office de la langue française. ${ }^{45}$

À l'époque, l'éditeur québécois avait refusé la version hexagonale du Bescherelle et retardé la publication du guide de conjugaison de quelques mois pour en retirer les éléments problématiques.

\section{Sous les vents de Neptune : le téléfilm}

L'aventure du roman policier de Fred Vargas ne s'est pas arrêtée à la version papier. Un téléfilm réalisé par Josée Dayan a été coproduit par la France et le Canada en 2007. Bien sûr, au Québec, la question de la langue s'est tout de suite posée : "Chez Transfilm, on assure que les dialogues en québécois colleront davantage à notre réalité que le langage à moitié inventé par Fred Vargas dans son roman. ${ }^{46}$ ”

L'adaptation du roman et des dialogues a été confiée à l'auteur Emmanuel Carrère qui a fait un travail remarquable. Par exemple, au moment où le policier québécois Aurèle Laliberté explique à son équipe comment il faudra travailler avec les Français, Fred Vargas a écrit :

Chacun de vous s'amanchera avec l'un des membres de la Brigade de Paris, et on changera les paires tous les deux ou trois jours. Allez-y de tout cœur mais menezles pas tambour battant pour vous faire péter les bretelles, ils ne sont pas infirmes des deux bras. Ils sont en période d'entraînement, ils s'initient. Alors formez-les au pas de grise pour commencer. Et faites pas de l'esprit de bottine s'ils ne vous comprennent pas ou s'ils parlent autrement que nous. Ils sont pas plus branleux que vous autres sous prétexte qu'ils sont français. ${ }^{47}$

Dans le téléfilm, ce passage est devenu:

À partir de demain, chacun de vous va travailler avec un Français. On va changer de paire à tous les deux jours pour ne pas prendre de mauvaises habitudes. Pour commencer, vous pouvez vous matcher avec qui vous voulez. J'veux pas de farce plate s'ils parlent différemment de nous autres ou ben s'ils vous comprennent pas. Sont pas plus niaiseux que nous autres parce qu'ils sont Français.

Autre exemple. Au moment où Adamsberg s'enfuit, parce qu'il est soupçonné de meurtre, la réplique : « Il a pris la quille de l'air, votre boss ! [...] Il nous a fait la passe de l'ours et il 
a pris le bord ${ }^{48} »$ devient, dans le téléfilm : « Votre boss nous a faite une crosse. Y'a sacré son camp le calisse. » Pas plus élégant, mais tellement plus québécois !

Le téléfilm en deux épisodes a été diffusé sur France 2 les 15 et 22 février 2008. Encore une fois, la critique française a été élogieuse pour Fred Vargas : « Un thriller haletant, parfois stressant et souvent drôle. Les échanges avec les policiers québécois sont savoureux et Vargas possède un vrai sens du dialogue, dont certains sont reproduits intégralement dans le film. Et ça sonne juste. ${ }^{49}$ "

Cependant, continuant sur sa série noire, l'histoire de Vargas n'a jamais été diffusée au Québec. Le diffuseur québécois a prétexté un manque de qualité du téléfilm : «On nous a vendu une série qui était extraordinaire, et à la livraison, excusez-moi, c'est une série qui n'est complètement pas à la hauteur de ce qui a été vendu. ${ }^{50}$ » Le coproducteur canadien a répliqué en dévoilant les problèmes financiers du diffuseur, qui s'apprêtait à déclarer faillite et qui tentait de ne pas payer son dû en dépréciant le téléfilm. L'histoire a fini en cour, sans que les Québécois puissent apprécier l'adaptation du roman qui les avait tant fait réagir.

\section{Conclusion}

Pour conclure, il est important de souligner que si le roman de Fred Vargas a eu tant d'échos, c'est que cette conception centralisatrice de la langue n'est plus de mise aujourd'hui. Les auteurs français qui font voyager leurs personnages au Québec s'associent généralement à des rédacteurs ou à des consultants québécois. Par exemple, un album de Lucky Luke a récemment été scénarisé par Laurent Gerra, qui a décidé de situer l'action au Québec:

Il trouvait logique que Lucky Luke, cow-boy créé par des francophones, plante un jour les talons de ses bottes dans cette «belle province » où l'on parle français. [...] Pour cela, de même que pour l'entrée en matière historico-comique et l'utilisation des expressions «typiques", Laurent Gerra a fait de la recherche. Il a lu, visité le musée de la Civilisation de Québec... et discuté avec sa «blonde », Lynda Lemay laquelle l'a bien aiguillé en matière de québécismes. Ainsi [...], pas de faux pas ici à la manière d'une Fred Vargas qui, à cause du pseudo joual qu'elle utilise dans Sous les vents de Neptune, a fait grincer bien des dents et des susceptibilités. ${ }^{51}$

De même, en 2006, deux illustrateurs français installés au Québec ont décidé de créer une bande dessinée qui se passerait dans le Québec des années 1920. Ils ont tout naturellement fait appel à un Québécois pour superviser les dialogues :

Quant à l'épineuse question de la langue, il apparaissait inévitable que ce soit quelqu'un d'ici qui supervise le tout. Afin de conférer aux dialogues une authenticité et d'éviter les erreurs qu'ont commises par le passé certains écrivains ayant choisi de camper leur histoire dans la Belle Province, Loisel et Tripp ont décidé de faire appel à Jimmy Beaulieu pour revoir les dialogues. ${ }^{52}$

À l'inverse, quand l'auteure québécoise Marie Laberge a choisi de situer une partie d'un de ses romans à Paris, elle a fait aussi appel à des réviseurs français :

Une gentille guéguerre de cultures est parfaite pour dérider le lecteur entre deux assassinats. Si on parvient à éviter les clichés... La délicieuse romancière française Fred Vargas, qui avait usé du procédé dans Sous les vents de Neptune (2004), dont l'action se déroule au Québec, s'était cassé les dents sur notre parlure. Pour s'assurer que l'accent français sonne vrai, Marie Laberge a fait relire Sans rien ni personne par trois amis de l'Hexagone..$^{53}$ 
La mésaventure linguistique de Sous les vents de Neptune de Fred Vargas apparaît donc comme un anachronisme au $\mathrm{xxI}^{\mathrm{e}}$ siècle, un cas tellement unique qu'il en est venu à symboliser l'erreur à ne plus commettre, confirmant ainsi l'avancée de la reconnaissance de l'autre, en France et au Québec.

\section{BIBLIOGRAPHIE}

Barbeau, Victor, Le français du Canada, Montréal, Les publications de l'Académie canadiennefrançaise, 1963.

Barbeau, Victor, Le Ramage de mon pays - le français tel qu'on le parle au Canada, Montréal, Éditions Bernard Valiquette, 1939.

Bergeron, Léandre, Dictionnaire de la langue québécoise, Montréal, VLB Éditeur, 1980.

Bescherelle, La Conjugaison pour tous, Paris, Hatier, 1997.

Cardinal, François, « Le joual de Fred Vargas à côté de la track », La Presse, Montréal, 25 avril 2004, « Lectures », p. 9.

Cloutier, Mario, «Un Fred Vargas en tournage au Québec », La Presse, Montréal, 21 septembre 2006, « Arts et Spectacles », p. 4.

Coppermann, Annie, « Le juge et le commissaire », Les Échos, 6 avril 2004, p. 19.

Delcroix, Olivier, « Fred Vargas : chercheuse le jour, romancière la nuit », Le Figaro, 27 avril 2004, p. 25.

Desmeules, Christian, « Vargas dans la fosse aux ours », Le Devoir, Montréal, 19 juin 2004, p. F8.

Dion, Jean, « Vous êtes formidables », Le Devoir, Montréal, 10 juillet 2004, p. B2.

Dionne, Narcisse-Eutrope, Le parler populaire des Canadiens français, Québec, Les Presses de l'Université Laval, 1974. Réimpression de l'édition originale de 1909.

Dupuis, Jérôme, « Qui est vraiment Fred Vargas », Lire, n 354, avril 2007, p. 32-35.

Gaillard, Roger, « Vargas, pelleteuse de nuages », Le Temps, 31 juillet 2004.

Gauvin, Lise, La fabrique de la langue, Paris, Éditions du Seuil, 2004.

Grisolia, Michel, « Sous les vents de Neptune : frissons au long cours façon Fred Vargas », L'Express, 5 avril 2004, p. 116.

Guéguen, Josiane, «Le souffle vivifiant de Fred Vargas, très noir ", Ouest-France, 28 mars 2004, p. 14.

Hausmann, Franz Josef « Les dictionnaires du français hors de France », dans Boisvert, Lionel, Claude Poirier et Claude Verreault (dir.), La lexicographie québécoise : bilan et perspectives, Québec, Les Presses de l'Université Laval, 1986, p. 3-19.

Larrivée, Pierre, « Les normes linguistiques et leur changement : la légitimation en cours du français québécois dans la littérature gallicane ", Langage et société, n 115, 2006, p. 103-127. 
Lebeau, Guillaume, Le mystère Fred Vargas, Paris, Éditions Gutenberg, 2009.

Lepage, Jocelyne, « Le cadeau de Fred Vargas », La Presse, Montréal, 4 juin 2006, « Arts et Spectacles », p. 11.

Lessard, Valérie, « Hull-Gatineau, "le trou du cul du monde" ? ", Le Droit, Ottawa-Gatineau, $1^{\mathrm{er}}$ mai 2004, p. A2.

L'Heureux, Serge, «Les démons du commissaire Adamsberg », Le Nouvelliste, Trois-Rivières, 12 juin 2004, p. 7.

Nicoud, Anabelle, « Christal Films à la recherche de capitaux », La Presse, Montréal, 13 mars 2008, «Arts et Spectacles », p. 1.

Patry, Richard, « Compte rendu du Dictionnaire de la langue québécoise, L. Bergeron, VLB éditeur, 1980 », Revue québécoise de linguistique, vol. 12, 1982, p. 209-216.

Saint-Hilaire, Mélanie, « Le loup dans Labergerie », L'Actualité, Canada, $1^{\mathrm{er}}$ novembre 2007, p. 108.

Sans auteur, « La musique de Vargas », Le Télégramme (Bretagne), 13 juin 2004.

Sarfati, Sonia, « Laurent Gerra signe Lucky Luke », La Presse, Montréal, 17 juillet 2004, « Arts et Spectacles », p. 1.

Séguin, Hubert, « Le Bescherelle nouveau s'en vient : ouatchez-vous ! », Le Devoir, Montréal, 17 décembre 1997, p. A9.

Seutin, Émile, André Clas et Manon Brunet, avec la collaboration de Marthe Faribault et Chantal Bouchard, Richesses et particularités de la langue écrite au Québec, Observatoire du français contemporain, département de linguistique et philologie, université de Montréal, 8 volumes, 1979-1982.

Société du parler français au Canada, Glossaire du parler français au Canada, Québec, les Presses de l'Université Laval, 1968. Réimpression de l'édition originale de 1930.

Steinlen, Claire, «France 2. Sous les vents de Neptune », Le Télégramme (Bretagne), 10 février 2008.

Sulte, Benjamin, La langue française en Canada, Lévis, Bibliothèque canadienne, Pierre-Georges Roy éditeur, 1898.

Tanguay, Antoine, « Loisel et Tripp accouchent d'une bande dessinée qui célèbre le Québec d'hier » Le Soleil, Québec, 14 avril 2006, p. B1.

Vargas, Fred, « Fred Vargas nous écrit », La Presse, Montréal, 11 juin 2006, « Arts et Spectacles », p. 13.

Vargas, Fred, Sous les vents de Neptune, éditions Viviane Hamy 2004, format J'ai lu policier, ${ }^{\circ}$ 8175, 2008.

\section{NOTES}

1. La notion de "variante nationale » repose sur la distinction faite par Franz Josef Hausmann: «On ne peut assigner au Québec, à la Belgique wallonne ou au Sénégal le statut de région au même titre qu'à l'Ouest de la France. De par leur souveraineté nationale, ces unités géolinguistiques méritent la dénomination de pays tout autant que la France. Les particularités lexicales de ces pays par rapport au français de France ne sont donc pas des régionalismes. Ils ne constituent pas une variante régionale de la langue française mais une variante nationale.» Franz 
Josef Hausmann, «Les dictionnaires du français hors de France », La lexicographie québécoise : bilan et perspectives, Québec, Presses de l'Université de Laval, 1986, p. 19. Dans cette optique, le concept de variation linguistique oppose les tenants d'une norme unique - et hexagonale - du français, aux défenseurs d'une norme interne propre à chaque variante nationale.

2. Notre acception de "francophone " inclut les Français. Dans cette optique, les écrivains français sont des écrivains francophones.

3. Dans l'emploi qu'en fait Lise Gauvin, la francophonie désigne l'ensemble des parlants français (langue première ou seconde) hors de France. Les écrivains français ne sont donc pas pour elle des écrivains francophones.

4. Lise Gauvin, La fabrique de la langue, Paris, Éditions du Seuil, 2004, p. 256.

5. Reconstitution des modes de vie humain et animal en fonction des artéfacts et ossements trouvés lors de fouilles.

6. Guillaume Lebeau, Le mystère Fred Vargas, Paris, Éditions Gutenberg, 2009, p. 51.

7. Roger Gaillard, «Vargas, pelleteuse de nuages », Le Temps, 31 juillet 2004.

8. «Ventes totales en France, poches compris (J'ai lu) : 5 millions d'exemplaires. [...] Sous les vents de Neptune en a totalisé 280 000. [...] Ses romans sont traduits dans 35 pays. » (Jérôme Dupuis, "Qui est vraiment Fred Vargas », Lire, avril 2007, p. 35)

9. Guillaume Lebeau, Le mystère Fred Vargas, op. cit., p. 324.

10. Roger Gaillard, «Vargas, pelleteuse de nuages », Le Temps, 31 juillet 2004.

11. Josiane Guéguen, «Le souffle vivifiant de Fred Vargas, très noir », Ouest-France, 28 mars 2004, p. 14.

12. Michel Grisolia, "Sous les vents de Neptune: frissons au long cours façon Fred Vargas ", L'Express, 5 avril 2004, p 116.

13. Annie Coppermann, « Le juge et le commissaire », Les Échos, 6 avril 2004, p. 19.

14. Guillaume Lebeau, Le mystère Fred Vargas, op. cit., p. 329.

15. François Cardinal, «Le joual de Fred Vargas à côté de la track», La Presse, 25 avril 2004, «Lectures », p. 9.

16. Jean Dion, «Vous êtes formidables ", Le Devoir, 10 juillet 2004, p. B2.

17. Valérie Lessard, « Hull-Gatineau, "le trou du cul du monde" ? , Le Droit, $1^{\mathrm{er}}$ mai 2004, p. A2.

18. Jocelyne Lepage, «Le cadeau de Fred Vargas », La Presse, 4 juin 2006, « Arts et Spectacles », p. 11.

19. Voir Lebeau, 2009, p. 133.

20. Olivier Delcroix, "Fred Vargas: chercheuse le jour, romancière la nuit», Le Figaro, 27 avril 2004, p. 25.

21. Fred Vargas, «Fred Vargas nous écrit », La Presse, 11 juin 2006, « Arts et Spectacles », p. 13.

22. Fred Vargas, Sous les vents de Neptune, Paris, J’ai lu policier, 2008, p. 437.

23. Ibid., p. 153.

24. Ibid., p. 186.

25. Christian Desmeules, «Vargas dans la fosse aux ours », Le Devoir, 19 juin 2004, p. F8.

26. Serge L'Heureux, « Les démons du commissaire Adamsberg », Le Nouvelliste, 12 juin 2004, p. 7.

27. Fred Vargas, Sous les vents de Neptune, op. cit., p. 132.

28. Ibid., p. 135.

29. Ibid., p. 136.

30. Ibid., p. 144.

31. Ibid., p. 176.

32. Ibid., p. 221.

33. Ibid., p. 132.

34. Ibid., p. 190.

35. Voir Seutin et al.

36. Fred Vargas, Sous les vents de Neptune, Paris, op. cit., p. 169. 
37. « un produit à la fois historique et synchronique, où se côtoient des mots récents, ou à tout le moins d'un usage très répandu en français québécois contemporain, et des mots vieillis, en désuétude, ou des mots provenant d'aires géographiques régionales très précises lorsqu'ils ne sont pas tout simplement des fossiles linguistiques totalement disparus du vocabulaire québécois d'aujourd'hui. [...] En bref, le Dictionnaire de la langue québécoise ne remplit convenablement à peu près aucune des exigences raisonnables que n'importe quel lecteur, qu'il soit spécialiste ou profane, est en droit d'attendre d'un dictionnaire." (Richard Patry, "Compte rendu du Dictionnaire de la langue québécoise ", Revue québécoise de linguistique, vol. 12, 1982, p. 211)

38. Ibid., p. 186.

39. Ibid., p. 269.

40. Ibid., p. 272.

41. Ibid., p. 185.

42. Ibid., p. 198.

43. Ibid., p. 279

44. Pierre Larrivée, "Les normes linguistiques et leur changement: la légitimation en cours du français québécois dans la littérature gallicane ", Langage et société, $\mathrm{n}^{\circ} 115,2006, \mathrm{p}$. 103-127.

45. Hubert Séguin, «Le Bescherelle nouveau s'en vient: ouatchez-vous!», Le Devoir, 17 décembre 1997, p. A9.

46. Mario Cloutier, «Un Fred Vargas en tournage au Québec », La Presse, 21 septembre 2006, " Arts et Spectacles », p. 4.

47. Fred Vargas, Sous les vents de Neptune, op. cit., p. 134.

48. Ibid., p. 269.

49. Claire Steinlen, «France 2. Sous les vents de Neptune», Le Télégramme (Bretagne), 10 février 2008.

50. Le diffuseur Christian Larouche, cité par Anabelle Nicoud, «Christal Films à la recherche de capitaux », La Presse, Montréal,13 mars 2008, « Arts et Spectacles », p. 1.

51. Sonia Sarfati, « Laurent Gerra signe Lucky Luke », La Presse, Montréal, 17 juillet 2004, « Arts et Spectacles », p. 1.

52. Antoine Tanguay, «Loisel et Tripp accouchent d'une bande dessinée qui célèbre le Québec d'hier » Le Soleil, Québec, 14 avril 2006, p. B1.

53. Mélanie Saint-Hilaire, « Le loup dans Labergerie », L'Actualité, $1^{\mathrm{er}}$ novembre 2007, p. 108.

\section{RÉSUMÉS}

Pour tout auteur, choisir d'écrire dans une langue ou dans la variante d'une langue qui n'est pas la sienne représente un défi de taille. Nous nous intéresserons au roman Sous les vents de Neptune, de l'écrivaine française Fred Vargas, qui a situé une partie de son intrigue au Québec, sans maîtriser la notion de variation linguistique. En analysant les réactions des lecteurs européens et québécois, de même que les caractéristiques de la langue québécoise de Vargas, nous aborderons la question de l'impérialisme linguistique et de son anachronisme au XXI ${ }^{\mathrm{e}}$ siècle.

For any author, it is not an easy thing to write using a language variation that is not his own. We will investigate Sous les vents de Neptune, a novel from the French writer Fred Vargas. A part of the story takes place in Québec, but the author is not familiar with the language variation used in this French province. We will analyse how European and Quebecer readers react to this novel and 
we will define what characterizes the language used by Vargas, which will allow us to approach the linguistic imperialism and its anachronism in the 21st century.

\section{AUTEUR}

NADINE VINCENT

Université de Sherbrooke (Québec) 